\title{
KDC Authentication for Secured Communication using Four-Way Handshake Protocol
}

\author{
lyswarya $\mathrm{R}$. \\ Assistant Professor \\ Department of Computer \\ Science and Engineering \\ Sri Venkateswara College \\ of Engineering
}

\author{
Muthunagai S.U. \\ Assistant Professor \\ Department of Computer \\ Science and Engineering \\ Sri Venkateswara College \\ of Engineering
}

\author{
Poorani S. \\ Assistant Professor \\ Department of Computer \\ Science and Engineering \\ Sri Venkateswara College \\ of Engineering
}

\author{
Anitha R., PhD \\ Professor and Head \\ Department of Computer \\ Science and Engineering \\ Sri Venkateswara College \\ of Engineering
}

\begin{abstract}
All the Wi-Fi connected devices are currently secured by WPA2 protocol but in recent studies it proves that the major vulnerability which exploits the man-in-the-middle attack it also referred as KRACK (Key Re-installation AttaCK). In this research, the improvisation of the current scheme of 4-way handshake in the WPA2 (Wi-Fi Protected Access-2) is discussed and has an advantage of already-in-use key exchanged in the handshake. The Key Distribution center (KDC) protocol improvises the WPA2 by randomly generating a session key per communication along with 4-way handshake key exchange in the protocol. Session key is used as a Pre- shared key for the derivation of further components in the key exchange. Thus, the confidentiality in the proposed protocol is maintained in secured manner.
\end{abstract}

\section{General Terms}

Security, Session Key, Access Point

\section{Keywords}

Key Distribution Center(KDC), KRACK(Key Re-installation AttaCK, Message Integrity Check (MIC)

\section{INTRODUCTION}

The current version of Wi-Fi uses the encryption protocol known as WPA-2 (Wi-Fi Protected Access 2) which is the enhanced and better version of its previous WPA [5]. WPA used 3-way handshake while WPA2 is different by applying 4-way handshake. Both the versions of Wi-Fi, Wi-FiPersonal and Wi-Fi- Enterprise, used in private and public networks respectively relies on the encryption protocol. The 4-way handshake is a key exchange protocol where the two parties agree on the key for further encryption of the messages transmitted between them. The key where they agree upon is called as Pr -Shared Key (PSK). Hence, the WPA2 is also referred as WPA2-PSK. WPA2 uses CCMP-AES (Counter Mode/CBC-MAC Protocol) Protocol as its security protocol. The size of the key used is 128 bits length.[5] Thus it provides and maintains both confidentiality and integrity. Although the security has been improvised, a recent flaw has made it vulnerable. The vulnerability exploits the confidentiality through man-in-the-middle attack [1]. The attack have to be at the range of the network and sniffs the packet through a software (which can be used for penetration testing).Wi-Fi does not have a reliable medium such as optical fibre cables as its [3] only medium is air. Here messages at the medium can be dropped easily, which makes the attack easily. The attacker requires repeated packet data to derive the key and hence penetrate the network [7]. This happens between the Access Point (AP) and any Wi-Fi supported devices (smart phone, laptops, PCs etc). The PSK which is installed at the end of exchange, the attacker captures it and these results in the AP to re-transmit the same key again which is referred as reauthentication phase [3]. During this phase, the attacker will attempt to capture the HMAC (Hashed Message Authentication Code) of the PSK and by using several reversed encryption process, the key is identified [2].

\section{RELATED WORK}

The data transferred via public Wi-Fi is not secured. WPA2 (Wi-Fi Protected Access-2) is a protocol which provides security for public Wi-Fi access. However, this protocol has vulnerability, which is a serious threat [1]. The messages from Wi-Fi modem/router drop in the medium while transmission, it can be eavesdropped and loses confidentiality and integrity [9]. The vulnerability in the algorithm gives the way for the middleman to gain access to the encrypted data. As a result, the middleman can control and modify the data in user's IOT devices. The authentication before the key exchange might enhance the confidentiality in the key [8] .

\subsection{Four-way Handshake}

The handshake is the mutual exchange of Pair-wise Master Key (PMK) and a session key called as Pair wise Transient Key. The handshake happens after when the client (any WPA2 supported device) is authenticated with the AP [3]. It consists of four messages passed over the WLAN. The first is given from AP called as Anonce (Authenticator nonce or number used once) [5]. On receiving the Anonce, the client computes from the parameters from PSK and derives PMK using Password-Based Key Derivation Function 2(PBKDF2).

\section{PMK=PBKDF2(PSK,SSID,SSID-Length,4096)}

The PMK is same as the PSK since there is no authentication server[6].With the PMK, PTK is again derived after client's confirmation of its authentication along with Snonce and MIC(Message Integrity Check).PTK derived by client is given to AP which further derives its own PTK. It extracts nonce received and generates $\mathrm{KCK}(\mathrm{Key}$ Conformation Key),KEK(Key Encryption Key),TCK()This MIC maintains the integrity in the exchange [5].

After generating the PTK, the AP checks MIC generated with the MIC received to ensure the integrity. GTK is also derived during this process. Then the AP transmits the PTK, GTK and permits the client to install the keys to its system [4]. 


\subsection{Key Re installation Attack}

The key re-installation attack occurs during the key exchange because the AP still accepts re-transmissions of keys, even when it is in the PTK acknowledgement state, the attacker can force a re-installation of the PTK. More precisely, he establishes a man-in-the-middle (MitM) [1] position between the supplicant and authenticator. This prevents the acknowledgement transferred. As a result, it will re-transmit key install message, which causes the AP to reinstall an already-in-use PTK. In turn, this resets the nonce being used by the data-confidentiality protocol [3]. Depending on which protocol is used, this allows an adversary to replay, decrypt, and/or forge packets.

Traffics in the air between a station and an AP can be eavesdropped by sniffing tools like wire shark. By using sniffing tools, attackers can capture data packets and get information from the packets. If an attacker analyzes packets that are not encrypted, it would be critical threat to users. Based on traffic analysis, other attacks can be tried such as Denial of Services (DoS), key recovery, fake authentication and Man in the Middle attack [1].

\section{PROPOSED METHODOLOGY}

The proposed architecture uses the Key Distribution Center (KDC) protocol. Since in the current WPA2 the authentication of client and server is not performed before the exchange, KDC authenticates only the client. This authentication is unique and random and is available only for the current session.

The use of a key distribution center is based on the use of a hierarchy of keys. At a minimum, two levels of keys are used. Communication between end systems is encrypted using a temporary key, often referred to as a session key. Typically, the session key is used for the duration of a logical connection, such as a frame relay connection or transport connection, and then discarded. Each session key is obtained from the key distribution center over the same networking facilities used for end-user communication. Accordingly, session keys are transmitted in encrypted form, using a master key that is shared by the key distribution center and an end system or user. For each end system or user, there is a unique master key that it shares with the key distribution center. Of course, these master keys must be distributed in some fashion. However, the scale of the problem is vastly reduced. If there are entities that wish to communicate in pairs, then, as was mentioned, as many as session keys are needed at any one time. However, only master keys are required, one for each entity. Thus, master keys can be distributed in some non cryptographic way, such as physical delivery.

\section{Algorithm for KDC:}

Input: Requesting key from KDC

Output: Session key generation

1. Compute A -> KDC:IDA $\|\mathrm{IDB}\| \mathrm{N}_{1}$

2. Authentication in KDC for both $A$ and $B$

$\mathrm{KDC} \rightarrow \mathrm{A}: \mathrm{E}\left(\mathrm{K}_{\mathrm{a},}\left[\mathrm{K}_{\mathrm{s}}\left\|\mathrm{ID}_{\mathrm{B}}\right\| \mathrm{N}_{1} \| \mathrm{E}\left(\mathrm{K}_{\mathrm{B},}\left[\mathrm{K}_{\mathrm{S}} \| \mathrm{ID}_{\mathrm{A}}\right]\right)\right]\right)$

3. $A->B: E\left(K_{b},\left[K_{b},\left[K_{S} \| I_{A}\right]\right)\right.$

4. B->A:E( $\left(\mathrm{K}_{\mathrm{S},}\left[\mathrm{N}_{2}\right]\right)$

5. A->B:E( $\left(\mathrm{K}_{\mathrm{S},}\left[\mathrm{F}\left(\mathrm{N}_{2}\right)\right]\right)$

When user A wishes to establish a logical connection with B and requires a one-time session key to protect the data transmitted over the connection. A has a master key, , known only to itself and the KDC; similarly, B shares the master key with the KDC. The following steps occur.

A issues a request to the KDC for a session key to protect a logical connection to B. The message includes the identity of $\mathrm{A}$ and $\mathrm{B}$ and $\mathrm{a}$ unique identifier,for this transaction, which we refer to as a nonce. The nonce may be a timestamp, a counter, or a random number; the minimum requirement is that it differs with each request. Also, to prevent masquerade, it should be difficult for an opponent to guess the nonce. Thus, a random number is a good choice for a nonce.

The KDC responds with a message encrypted using. Thus, A is the only one who can successfully read the message, and A knows that it originated at the KDC.

A stores the session key for use in the upcoming session and forwards to $\mathrm{B}$ the information that originated at the KDC for $\mathrm{B}$, namely, $\mathrm{E}(\mathrm{Kb},[\mathrm{Ks} \| \mathrm{IDA}])$. Because this information is encrypted with, it is protected from eavesdropping. B now knows the session key, knows that the other party is A (from IDA ), and knows that the information originated at the KDC (because it is encrypted using ).

The proposed model uses the session key generated during the first receipt of client to AP. The session key is 6-bit randomly generated key which is further used as the PSK and added to the passphrase. And this key is allowed only for the current session. Once the session is done, the key is eliminated.

Metrics comparison is done between WPA3 and KDC protocol such that KDC provides acceptable authentication before the handshake.

A Python program is implemented to demonstrate the working of the model. 


\section{SYSTEM ANALYSIS}

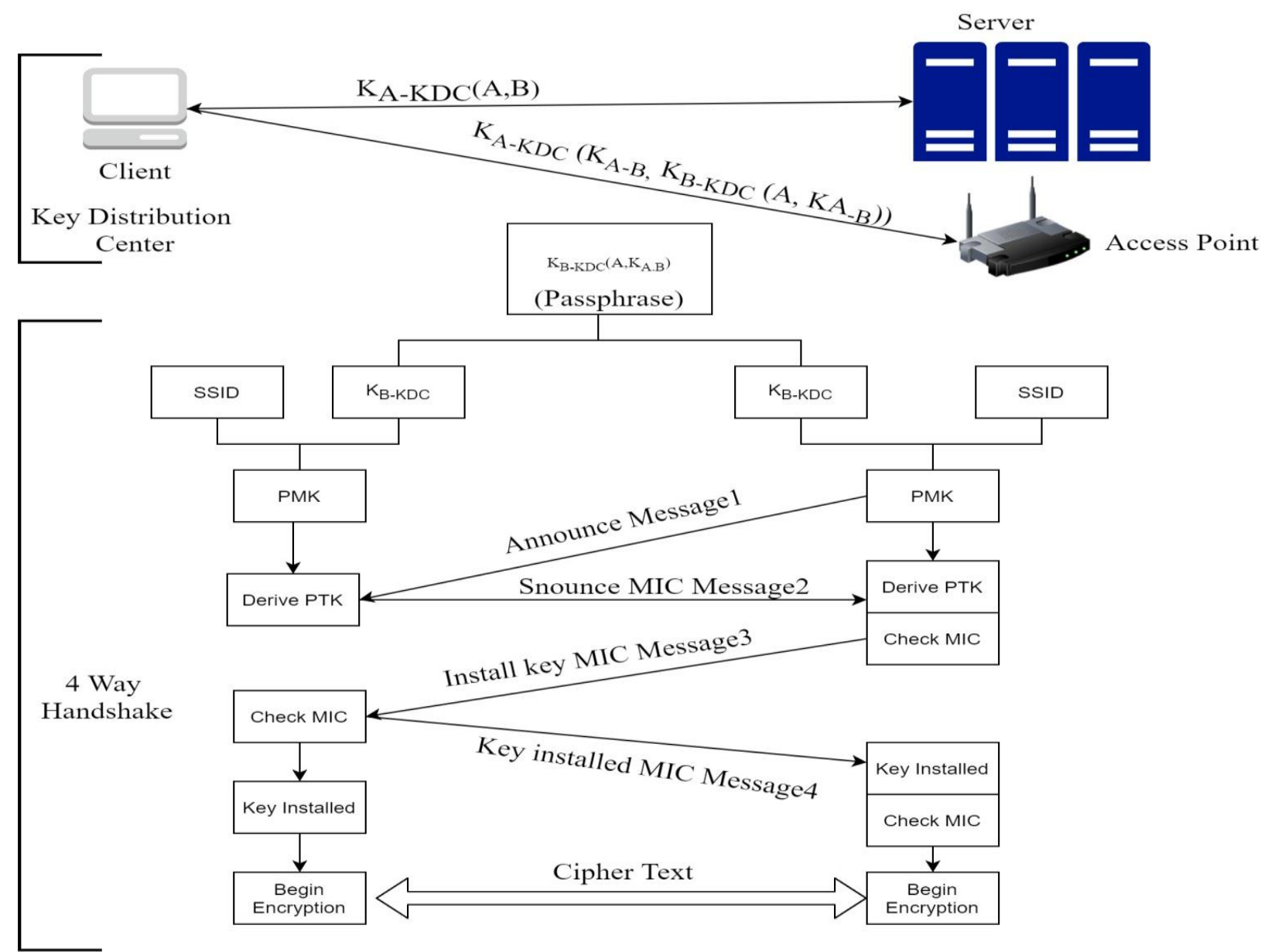

Fig 1 : Proposed architecture

\subsection{MakePRF function:}

This function is a pseudo random function used to generate the PTK.

def PRF (key,A,B)

nbyte $=64$

$\mathrm{i}=0$

$\mathrm{R}=\mathrm{b}$

While $(\mathrm{i}<=(($ nbyte $* 8+159) / 160))$;

hmacsha1 $=$ hmac.new $\left(\right.$ key, $\mathrm{A}+\operatorname{chr}\left(0^{*} 00\right)$.encode ()$+3+\operatorname{chr}(\mathrm{I})$.en code(),sha1)

$\mathrm{P}=\mathrm{R}+\mathrm{hmacsha1}$.digest ()

$\mathrm{i}=\mathrm{i}+1$;

return $\mathrm{R}[0:$ nbyte]

\subsection{MakeAB function}

It generates the parameters required for PTK. These are Anonce, Snonce, client and AP's MAC addresses.

def make $\mathrm{AB}$ (anonce,snonce,apmac,climac)

$\mathrm{A}=\mathrm{b}$ "pairwise key expansion"

$\mathrm{B}=(\min (\operatorname{apmac}, \operatorname{climac})+\max ($ apmac, $\operatorname{climac})+\min ($ $\operatorname{return}(\mathrm{A}, \mathrm{B})$

\subsection{MakeMIC function}

It generates the Message Integrity Check (MIC) to ensure the unauthorized message modification.

def makemic(pwd,SSID,A,B, data,wpa = false):

Pmk=pbkdf2 hmac('sha1',pwd.encode('ascii'),ssid.encode('a scii'),4096,32)

$\mathrm{ptk}=\mathrm{PRF}(\mathrm{pmk}, \mathrm{A}, \mathrm{B})$

hmacfunc $=$ md 5 if wpa else sha 1

mics $=[$ hmac.new $(\mathrm{ptk}[0: 16], \mathrm{I}, \mathrm{hmacFunc}) \cdot \operatorname{digest}()$

for I in data]

return (mics,ptk,pmk)

\subsection{KDC function}

This is the proposed scheme implemented as a function.

def $k d c(m s g 1, i d a)$ :

if(ida=="thisisida"):

$\mathrm{ks}=\operatorname{str}(\operatorname{random} \cdot \operatorname{randint}(10000000,19999999))$

$\operatorname{msg} 2=m s g 1+\mathrm{ks}$

return msg2

This function first authenticates and verifies the client and after verification sends the PSK and concatenates it with request message. The client must separate the key and further use it for derivation. 
The below two screenshots described the output with using the KDC authentication protocol and without using the authentication protocol.

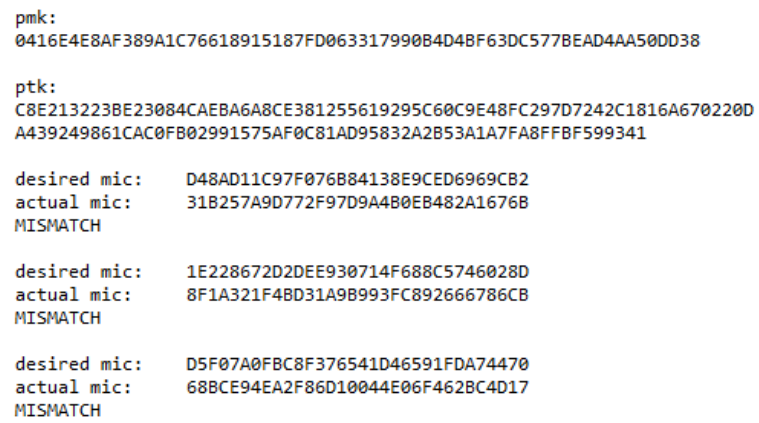

Fig 2: Output when KDC is applied

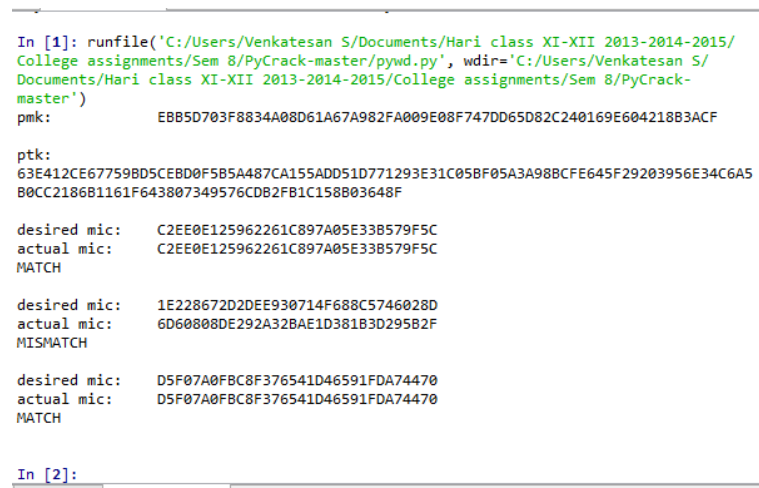

Fig 3: Output when KDC is not applied

Table 1: WITHOUT KDC

\begin{tabular}{|c|c|c|c|}
\hline Session & Desired MIC & Actual MIC & Integrity \\
\hline 1 & $\begin{array}{l}\text { C2EE0E1259 } \\
62262 \mathrm{C} 897 \mathrm{~A} \\
05 \mathrm{E} 33 \mathrm{~B} 579 \mathrm{~F} \\
5 \mathrm{C}\end{array}$ & $\begin{array}{l}\text { C2EE0E1259 } \\
\text { 62262C897A0 } \\
\text { 5E33B579F5 } \\
\text { C }\end{array}$ & MATCHED \\
\hline 2 & $\begin{array}{l}\text { 1E228672D2 } \\
\text { DEE930714F } \\
688 C 5746028 \\
\text { D }\end{array}$ & $\begin{array}{l}\text { 6D60808DE2 } \\
\text { 92A32BAE1 } \\
\text { D38183D295 } \\
\text { B2F }\end{array}$ & MISMATCHED \\
\hline 3 & $\begin{array}{l}\text { DSF07A0FB } \\
\text { C8F376541D } \\
46591 F D A 74 \\
470\end{array}$ & $\begin{array}{l}\text { DSF07A0FB } \\
\text { C8F376541D } \\
\text { 46591FDA74 } \\
470\end{array}$ & MATCHED \\
\hline
\end{tabular}

Table 2: WITH KDC

\begin{tabular}{|c|c|c|c|}
\hline Session & Desired MIC & Actual MIC & Integrity \\
\hline 1 & $\begin{array}{l}\text { D48A11C97F07 } \\
\text { 6B84138E9CED } \\
\text { 6969CB2 }\end{array}$ & $\begin{array}{l}\text { 31B257A9D } \\
\text { 772F97D9A } \\
\text { 4BOEB482 } \\
\text { A1676B }\end{array}$ & MISMATCHED \\
\hline 2 & $\begin{array}{l}\text { 1E228672D2DE } \\
\text { E930714F68865 } \\
\text { 746028D }\end{array}$ & $\begin{array}{l}\text { 8F1A321F4 } \\
\text { BD31A9B9 } \\
\text { 93FC89266 } \\
6786 \mathrm{CB}\end{array}$ & MISMATCHED \\
\hline 3 & $\begin{array}{l}\text { D5F07A0FBC8F } \\
376541 \mathrm{D} 46591 \mathrm{~F} \\
\text { DA74470 }\end{array}$ & $\begin{array}{l}\text { 68BCE94E } \\
\text { A2F86D100 } \\
\text { 44E06F462 } \\
\text { BC4D17 }\end{array}$ & MISMATCHED \\
\hline
\end{tabular}

Table 3 : Metrics Comparisons between WPA3 and KDC

\begin{tabular}{|c|c|c|}
\hline Attack & WPA3 & KDC \\
\hline De-authentication & Yes & Yes \\
\hline $\begin{array}{c}\text { Handshake capture } \\
\text { dictionary }\end{array}$ & Yes & Yes \\
\hline $\begin{array}{c}\text { PMKID Hash } \\
\text { dictionary Attack }\end{array}$ & Yes \\
\hline $\begin{array}{c}\text { Rough Access } \\
\text { point }\end{array}$ & Partial & Fully \\
\hline $\begin{array}{c}\text { Handshake Capture } \\
\text { En/Decryption }\end{array}$ & Yes & Yes \\
\hline $\begin{array}{c}\text { KRACK } \\
\text { authentication }\end{array}$ & Yes & Yes \\
\hline
\end{tabular}

\section{CONCLUSION AND FUTURE WORK}

The proposed scheme provides the improvisation of the current WPA2 security protocol in Wi-Fi networks. The key generated from the protocol is random and temporary. This makes it impossible for derivation of messages using key obtained by packet sniffing. Without KDC the Desired MIC and Actual MIC will be matched so that packet sniffing can be done easily.But with KDC the Desired MIC and Actual MIC will not be matched so that packet sniffing is impossible. After implementing KDC parameters like Rough Access Point is achieved fully and Before Handshake Authentication has been successfully implemented for the packet sniffing attack as shown in fig

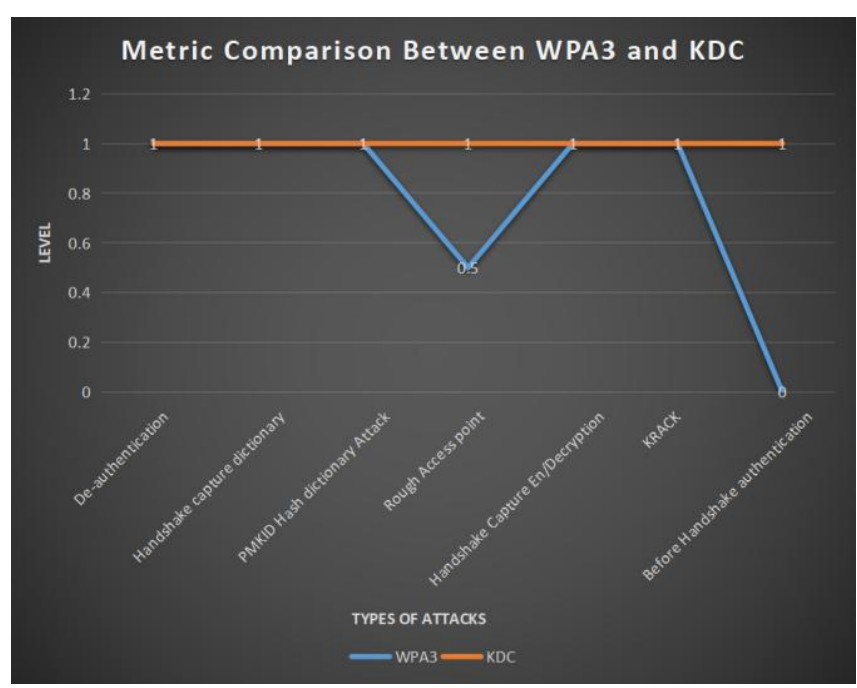

Fig 4: Metric Comparison Between WPA3 and KDC

In future, the scope of this scheme can give random session key for each key exchange.

\section{ACKNOWLEDGMENTS}

We would like to express our special thanks of gratitude to our institution who helped us to complete the paper. 


\section{REFERENCES}

[1] Agarwal M, Biswas S,and Nandi S, Apr. (2015).'Advanced stealth man-in-the-middle attack in WPA2 encrypted Wi-Fi networks,'IEEE Commun. Lett., Vol. 19, No. 4, pp. 581-584

[2] Bai Qinghai, Zhang Wenbo, Jiang Peng, LU Xu ,(2012) , 'Research on Design Principles of Elliptic Curve Public Key Cryptography and Its Implementation' IEEE Explore ,pp 3-5.

[3] Ghanem M.C and Ratnayake D.N , (2016),'Enhancing WPA2- PSK four-way handshaking after reauthentication to deal with de-authentication followed by brute-force attack a novel re- authentication protocol' in Proc. International Conference. Cyber Situational Awareness, Data Analyst Assessment (CyberSA), London, U.K.,pp. 1-7.

[4] Ghilen, M. Azizi, and Bouallegue R, (2015), 'Integration of a quantum protocol for mutual authentication and secret key distribution within 802.11i standard' in Proc. IEEE/ACS International Conference Computer System Application. (AICCSA), Marrakech, Morocco, pp. 1-7.
[5] Jae Won Noh, Jeehyeong Kim And Sung Yun Cho, (2018) ' Secure Authentication and Four-Way Handshake Scheme for protected Individual Communication in Public Wi- Fi Networks' in IEEE Explore.

[6] Liu Y, Wang Y, and Chang G , (2017), 'Efficient privacy- preserving dual authentication and key agreement scheme for secure $\mathrm{V} 2 \mathrm{~V}$ communications in an IoV paradigm,' IEEE Trans. Intell. Transp. System, Vol. 18, No. 10, pp. 2740-2749.

[7] Mitchell J.C and C. He, (2005), 'Security analysis and improvements for IEEE 802.11i' ,in Proc. Network Distributed System Security Symptoms (NDSS), San Diego, CA, USA, pp. 90-110.

[8] Noh J, Kim J, Kwon G, and Cho S , (2016), 'Secure key exchange scheme for WPA/WPA2-PSK using public key cryptography,' in Proc. IEEE International Conference Consum. Electronic-Asia (ICCE), Seoul, South Korea, pp.1-4.

[9] Subhas Barman,Samiran Chattopadhyay,Debasis Samanta,(2015) 'An Approach to Cryptographic Key Distribution Through Fingerprint Based Key Distribution Centre' IEEE.pp3-4. 